\title{
Seismic Response Analysis for Ordinary State-Based Peridynamics in a Linear Isotropic Elastic Material
}

\author{
Taiki Shimbo ${ }^{1} \cdot$ Ryota Itto ${ }^{1} \cdot$ Koutaro Inaba ${ }^{1} \cdot$ Kouichi Araki $^{2} \cdot$ Naoto Watanabe $^{3}$
}

Received: 19 July 2019 / Accepted: 2 February 2020 / Published online: 7 March 2020

(C) The Author(s) 2020

\begin{abstract}
Earthquakes may induce the failure of soil-structures built with a geo-material. It is therefore a crucially important aspect to study how a crack initiates and propagates in these soil-structures to analyze and improve their earthquake resistance. Peridynamics, although a powerful method for such an analysis, has not been applied to seismic response analysis of soil-structures on a viscous boundary surface that can consider an input seismic wave. To apply seismic response analysis to ordinary state-based peridynamics (OSB-PD), this paper proposed a fictitious viscous boundary layer and Rayleigh damping components constructed with time derivative of force density vectors. OSB-PD was semi-verified through the comparison of calculated response acceleration via the proposed method and via the dynamic finite element method (FEM). The discussions were stressed on the effect of Poisson's ratio, the frequency, and the Rayleigh damping components relative to the calculated response acceleration in both methods. Moreover, the seismic response acceleration at the top of the analysis model was calculated through the proposed method using an actual seismic acceleration time history, and then the result was compared with that calculated by the dynamic FEM. The results of both methods in the seismic response analysis agreed well with each other. From these results, we verified that the fictitious viscous layer can represent incoming/outgoing acceleration wave from the bottom of the analysis domain, and that Rayleigh damping can be expressed using the time derivative of force density vectors.
\end{abstract}

Keywords Seismic response analysis · Ordinary state-based peridynamics $\cdot$ Fictitious viscous layer $\cdot$ Rayleigh damping components

Taiki Shimbo

shimbo@ishikawa-nct.ac.jp

1 National institute of technology, Ishikawa College, Kitacyujo, Tsubata, Ishikawa 929-0392, Japan

2 GODAI KAIHATSU Corporation, 1-35 Kuroda, Kanazawa City, Ishikawa 921-8051, Japan

3 KFC LTD, 11-5 Yadaminami, Higashi-ku, Nagoya City, Aichi 461-0048, Japan 


\section{Introduction}

Over the recent years, Japan has been visited and plagued by several earthquakes, such as the Tohoku Earthquake off the Pacific coast in 2011, the Kumamoto Earthquake in 2016, and the Hokkaido Eastern Iburi Earthquake and Osaka Earthquake in 2018. Earthquakes may induce failure of an embankment, a fill-dam, railroad, and other soilstructures. Several dynamic finite element method (dynamic FEM) codes have been used in the region to predict the deformation of embankments [1-4]. These codes, in particular, do not only analyze response acceleration and finite strain, but also the liquefaction phenomenon in soil-structure. Moreover, these codes are very useful for evaluating the earthquake resistance of soil-structures, but do not treat the initiation and propagation of cracks in soils. Understanding the nature of cracks is essential to expressing the occurrence and progress of failure; therefore, their initiation and propagation should be further understood to improve the earthquake resistance of the soil-structure. We have recently reported on a seismic response analysis method using X-FEM on a viscous boundary surface with arbitrary interfaces [5], which can calculate the residual displacement of a sliding surface (interface) in an embankment caused by an earthquake. With the method, however, there were many problems associated with a Mode-II crack propagation analysis under a compressive loading condition (e.g., location of a crack initiation, crack propagation criteria, fracture toughness of sand/clay). On this basis, the Mode-II crack propagation process was not considered in that report. The difficulties encountered in the Mode-II crack propagation under the compressive loading condition utilizing the classical continuum mechanics can be overcome with peridynamics (PD) [6-8].

PD is a nonlocal formulation theory of continuum mechanics introduced by Silling [9] initially to solve discontinuous problems. Silling specifically introduced the bond-based PD (BB-PD), which permits the initiation and propagation of a crack. Nevertheless, as a bond is independently related to other bonds, an arbitrary Poisson's ratio cannot be expressed by BBPD. To overcome this limitation, Silling et al. proposed the state-based PD (SB-PD) [10]. SBPD is categorized into two types, namely, the ordinary state-based PD (OSB-PD) and the nonordinary state-based PD (NSB-PD). Presently, there has been an increasing interest to study PD, especially with respect to Mode-II shear crack [6-8] and elastic-plastic material [11-13], PD and FEM coupling [14-16], simulation of hydraulic fracture [17-20], simulation of the pre-notched gravity dam [21], and so on [22-25].

Wang et al. [26] presented the usefulness of a transmitted boundary condition for onedimensional PD. The boundary condition can remove undesired reflections as traveling waves are allowed to pass through the boundary and an incident wave to enter the computational domain. However, the coefficient of the boundary condition is dependent on wave numbers; in general, a seismic wave has several wave numbers. Lysmer and Kuhlemeyer [27] formulated a viscous boundary that is independent of wave number. The boundary cannot input displacement condition but can express incoming/outgoing acceleration waves. Nonetheless, such viscous boundary has not been applied to OSBPD yet. Meanwhile, in seismic response analysis, a material damping is necessary for considering the response acceleration of soil-structure. In this paper, we introduce a fictitious viscous boundary layer, referring to Lysmer and Kuhlemeyer, and Rayleigh damping components for the OSB-PD. To semi-verify the proposed method, we compare the response acceleration calculated by the proposed method to that calculated by dynamic FEM, along with the effect of Poisson's ratio, input seismic wave number, 
and Rayleigh damping component. Finally, we compare a seismic response analysis with the proposed method and dynamic FEM, using an actual seismic acceleration time history.

\section{Formulation of Seismic Response Analysis in a Linearly Isotropic Elastic Material}

\subsection{Definition of a Semi-Infinite Domain}

Figure 1 shows that relationship between a seismic wave and a one-dimensional domain, with the behavior of seismic waves particularly in focus. Here a seismic wave first enters from the bottom of the domain and travels upwards. As soon as it reaches the ground surface, the seismic wave is then reflected and travels downwards. Finally, the seismic wave reaches the bottom of the domain and exits from the domain. Note that the seismic wave separates with an incident wave (E-wave) traveling upwards and a reflected wave (F-wave) traveling downwards, which may combine together to form an $\mathrm{E}+\mathrm{F}$ wave. Moreover, note that an incoming wave indicates that the seismic wave enters from the bottom of the domain, whereas an outgoing wave indicates its exit from the domain.

Consider a two-dimensional semi-infinite elastic body $\Omega$, as shown in Fig. 2. It is necessary to calculate the semi-infinite region in which $\Omega$ has two types of fictitious boundaries: a vertical boundary and horizontal boundary, which can express an incoming/outgoing wave. In the vertical boundary, there are many types of the boundaries (e.g., viscous boundary, vertical fixed boundary). To use a viscous vertical boundary in $\Omega$, a viscous force of a one-dimensional analysis on out-side $\Omega$ is required. It is not easy to apply the viscous force to a two-dimensional boundary because of the number of particles within $\delta$ is lack near the boundary. Therefore, in this paper, for easy implementation, the vertical boundary defines a fictitious vertical displacement boundary $\Gamma_{u}^{v}$. In addition, the horizontal boundary defines a fictitious horizontal viscous boundary $\Gamma_{\text {vis }}^{h}$, in which $\Gamma_{\text {vis }}^{h}$ indicates an input seismic wave surface. Considering a viscous boundary $\Gamma_{\text {vis }}^{h}$ can express an incoming/outgoing wave. In doing so [27], a viscous force is necessary to act on $\Gamma_{\text {vis }}^{h}$. Moreover, when the pressure-wave velocity is $V_{\mathrm{p}}:=\sqrt{\rho / \kappa}$ and

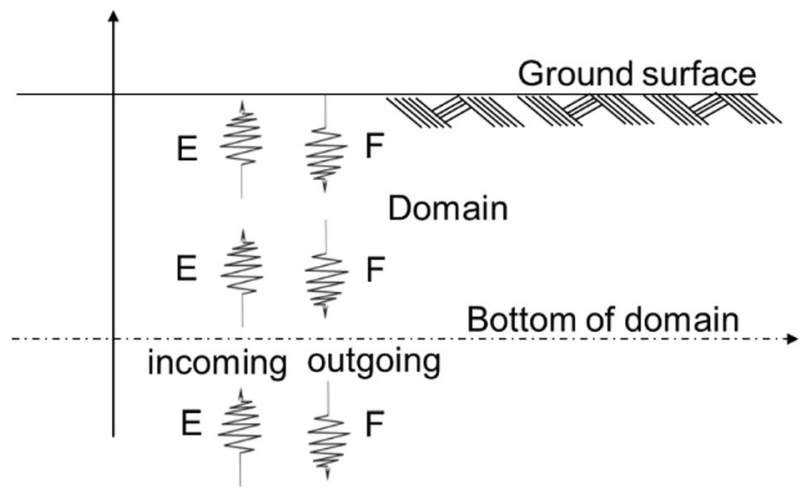

Fig. 1 Relationship between seismic wave and domain 


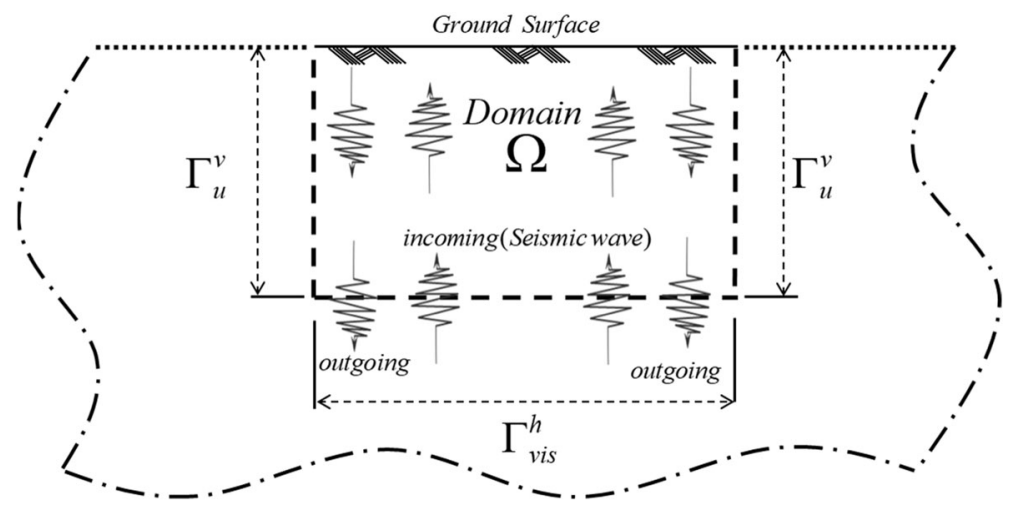

Fig. 2 Definition of a semi-infinite domain $\Omega$

the shear-wave velocity is $V_{\mathrm{s}}:=\sqrt{\rho / \mu}$, a viscous force assumes the Cartesian coordinates (i.e., $x$ and $y$ indicate the horizontal and vertical directions, respectively):

$$
\left\{\begin{array}{l}
f_{\text {vis }, x} \\
f_{\text {vis }, y}
\end{array}\right\}=-\rho\left[\begin{array}{cc}
V_{\mathrm{s}} & 0 \\
0 & V_{\mathrm{p}}
\end{array}\right]\left\{\begin{array}{l}
\dot{u}_{x} \\
\dot{u}_{y}
\end{array}\right\}
$$

where $\rho, \mu$, and $\kappa$ are respectively, the material density, shear modulus, and bulk modulus and; $\dot{u}_{x}$ and $\dot{u}_{y}$ represent the velocity components of $\Gamma_{\mathrm{vis}}^{h}$. A viscous force is a term to indicate the theoretical traction force on the surface $\Gamma_{\text {vis }}^{h}$, caused by E $+\mathrm{F}$ waves. It is obtained assuming that the material under $\Gamma_{\text {vis }}^{h}$ is a linear elastic material with shear velocity $V_{\mathrm{s}}$.

For simplicity of discussions, as with many seismic response analyses in a two-dimensional model, only horizontal viscous force is considered in this paper, that is, only the horizontal viscous force by $\dot{u}_{x}$ is used and the vertical displacement $u_{y}$ is constrained.

\subsection{Peridynamic Equation of Motion for Seismic Responses Analysis}

The peridynamic equation of motion introduced by Silling [9]. In this paper, we adopted the OSB-PD by Madenci and Oterkus [11]. The discretized state-based peridynamics form is

$$
\ddot{\rho} \mathbf{u}\left(\mathbf{x}_{i}, t\right)=\sum_{j=1}^{N_{H}}\left\{\underline{\mathbf{T}}\left(\mathbf{x}_{i}, t\right)\left\langle\mathbf{x}_{j}-\mathbf{x}_{i}\right\rangle-\underline{\mathbf{T}}\left(\mathbf{x}_{j}, t\right)\left\langle\mathbf{x}_{i}-\mathbf{x}_{j}\right\rangle\right\} d V_{j}+\mathbf{b}\left(\mathbf{x}_{\mathbf{i}}, \mathbf{t}\right)
$$

where $t, N_{H}, \mathbf{x}_{i}, j, \boldsymbol{u}, V_{j}$, and $\mathbf{b}$ are a calculation time, number of material points in a horizon, coordinate of a material point $i$, other material points in a horizon, a displacement, an incremental volume, and a body force, respectively.

In the case of OSB-PD utilizing an isotropic linear elastic material, Madenci and Oterkus [22], Madenci and Oterkus [11], Chen et al. [12], and subsequent studies demonstrated a force density vector $\underline{\mathbf{T}}\left(\mathbf{x}_{i}, t\right)\left\langle\mathbf{x}_{j}-\mathbf{x}_{i}\right\rangle$ as

$$
\underline{\mathbf{T}}\left(\mathbf{x}_{i}, t\right)\left\langle\mathbf{x}_{j}-\mathbf{x}_{i}\right\rangle=\frac{1}{2} A \frac{\mathbf{y}_{j}-\mathbf{y}_{i}}{\left|\mathbf{y}_{j}-\mathbf{y}_{i}\right|}
$$

where 


$$
\begin{aligned}
& A=4\left(\delta b s_{i j}+a d \omega_{i j} \Lambda_{i j} \theta_{i}\right), \\
& \theta_{i}=d \delta \sum_{j=1} s_{i j} \Lambda_{i j} V_{j}, \\
& \Lambda_{i j}=\frac{\mathbf{y}_{j}-\mathbf{y}_{i}}{\left|\mathbf{y}_{j}-\mathbf{y}_{i}\right|} \cdot \frac{\mathbf{x}_{j}-\mathbf{x}_{i}}{\left|\mathbf{x}_{j}-\mathbf{x}_{i}\right|}, s_{i j}=\frac{\left|\mathbf{y}_{j}-\mathbf{y}_{i}\right|-\left|\mathbf{x}_{j}-\mathbf{x}_{i}\right|}{\left|\mathbf{x}_{j}-\mathbf{x}_{i}\right|}, \omega_{i j}=\frac{\delta}{\left|\mathbf{x}_{j}-\mathbf{x}_{i}\right|}, \\
& a=\frac{1}{2}(\kappa-2 \mu), b=\frac{6 \mu}{\pi \bar{h} \delta^{4}}, d=\frac{2}{\pi \bar{h} \delta^{3}},
\end{aligned}
$$

where $\mathbf{y}_{i}$ and $\mathbf{y}_{j}$ are deformed position of particles $\mathbf{x}_{i}, \mathbf{x}_{j} ; s, \omega, \bar{h}, \delta$, and $\theta_{i}$ are stretch between two material points, influence function, thickness of an analysis model, a radius of a horizon, and a dilatation term of material point $i$, respectively; $a, b$, and $d$ are material parameters.

Considering a seismic wave entering a body $\Omega$ from a horizontal viscous boundary $\Gamma_{\text {vis }}^{h}$, the already-known displacement $\mathbf{u}_{g}$ (i.e., caused by a seismic wave) and the relative displacement $\mathbf{u}_{r}$ can be assumed. Therefore, the unknown displacement is given by $\mathbf{u}=\mathbf{u}_{r}+\mathbf{u}_{g}$. By substituting $\mathbf{u}$ into Eq. (2), the equation can be divided into already-known quantities and unknown relative quantities:

$$
\begin{array}{r}
\rho\left(\ddot{\mathbf{u}} r\left(\mathbf{x}_{i}, t\right)+\ddot{\mathbf{u}}_{g}\left(\mathbf{x}_{i}, t\right)\right)=\sum_{j=1}^{N_{H}}\left\{\underline{\mathbf{T}}\left(\mathbf{x}_{i}, t\right)\left\langle\mathbf{x}_{j}-\mathbf{x}_{i}\right\rangle-\underline{\mathbf{T}}\left(\mathbf{x}_{j}, t\right)\left\langle\mathbf{x}_{i}-\mathbf{x}_{j}\right\rangle\right\} d V_{j}+\mathbf{b}\left(\mathbf{x}_{\mathbf{i}}, \mathbf{t}\right) \\
\therefore \rho \ddot{\mathbf{u}}_{\mathbf{r}}\left(\mathbf{x}_{\mathbf{i}}, \mathbf{t}\right)=\sum_{\mathbf{j}=\mathbf{1}}^{\mathbf{N}_{\mathbf{H}}}\left\{\underline{\mathbf{T}}\left(\mathbf{x}_{\mathbf{i}}, \mathbf{t}\right)\left\langle\mathbf{x}_{\mathbf{j}}-\mathbf{x}_{\mathbf{i}}\right\rangle-\underline{\mathbf{T}}\left(\mathbf{x}_{\mathbf{j}}, \mathbf{t}\right)\left\langle\mathbf{x}_{\mathbf{i}}-\mathbf{x}_{\mathbf{j}}\right\rangle\right\} \mathbf{d} \mathbf{V}_{\mathbf{j}}+\mathbf{b}\left(\mathbf{x}_{\mathbf{i}}, \mathbf{t}\right)-\rho \ddot{\mathbf{u}}_{\mathbf{g}}\left(\mathbf{x}_{\mathbf{i}}, \mathbf{t}\right)
\end{array}
$$

Because the already-known displacement $\mathbf{u}_{g}$ is a rigid motion, it does not induce any force density vectors $\underline{\mathbf{T}}\left(\mathbf{x}_{i}, t\right)\left\langle\mathbf{x}_{j}-\mathbf{x}_{i}\right\rangle$ and $\underline{\mathbf{T}}\left(\mathbf{x}_{j}, t\right)\left\langle\mathbf{x}_{i}-\mathbf{x}_{j}\right\rangle$. Consequently, the unknown displacement is only the relative displacement $\mathbf{u}_{r}$. Accordingly, $\mathbf{u}_{r}$ will be described as unknown displacement $\mathbf{u}$ in the succeeding discussions.

\subsection{Rayleigh Damping Components}

For material damping, Rayleigh damping components were considered in this paper. Basically, Rayleigh damping has two types: mass-proportional damping and stiffness-proportional damping. In the classical continuum mechanics of an isotropic linear elastic material, these are respectively denoted by the symbols $\alpha$ and $\beta$.

The Rayleigh damping components can be assumed to be equivalent to the following equilibrium equation in the classical continuum mechanics:

$$
\rho \ddot{\mathbf{u}}+\alpha \rho \dot{\mathbf{u}}=\operatorname{div} \boldsymbol{\sigma}+\beta \operatorname{div} \dot{\boldsymbol{\sigma}}+\mathbf{b}-\rho \mathbf{u}_{g} \quad \text { for } \mathbf{x} \in \Omega
$$

which leads to the following well-known MCK-type finite element discretized equations of motion:

$$
[\mathbf{M}]\{\ddot{\mathbf{d}}\}+[\mathbf{C}]\{\dot{\mathbf{d}}\}+[\mathbf{K}]\{\mathbf{d}\}=\left\{\mathbf{F}^{\text {ext }}\right\} .
$$

Where $\sigma$ is stress, $[\mathbf{M}]$ is mass matrix, and $[\mathbf{K}]$ is stiffness matrix of an isotropic linear elastic material; $[\mathbf{C}]:=\alpha[\mathbf{M}]+\beta[\mathbf{K}]$ is Rayleigh damping matrix; $\left\{\mathbf{F}^{\mathrm{ext}}\right\}$ is an external force that includes body force and an inertial force induced by a seismic acceleration and; $\{\mathbf{d}\},\{\mathbf{d}\}$, and $\{\mathbf{d}\}$ represent the nodal acceleration, nodal velocity, and nodal displacement, respectively. 


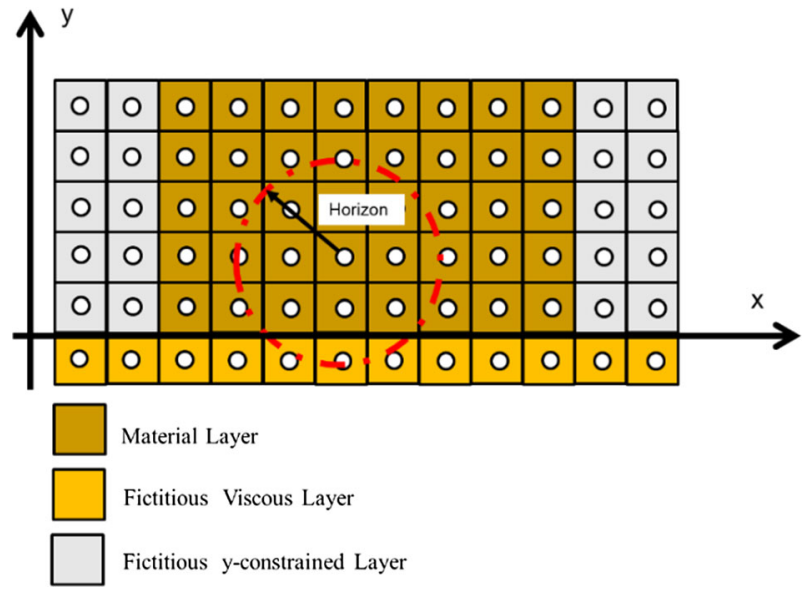

Fig. 3 Definition of layers in the analysis model

Comparatively, for Eqs. (5) and (6), a mass-proportional term and a stiffness-proportional term for OSB-PD are required to introduce Rayleigh damping components into Eq. (5). Therefore, with reference to Eq. (6), the mass-proportional term can be defined as,

$$
\rho \alpha \dot{\mathbf{u}}\left(\mathbf{x}_{i}, t\right) .
$$

In addition, the stiffness-proportional term is defined by a time derivative of force density vectors on the right-hand side of Eq. (5) as follows:

$$
\beta \frac{d}{d t}\left[\sum_{j=1}^{N_{H}}\left\{\underline{\mathbf{T}}\left(\mathbf{x}_{i}, t\right)\left\langle\mathbf{x}_{j}-\mathbf{x}_{i}\right\rangle-\underline{\mathbf{T}}\left(\mathbf{x}_{j}, t\right)\left\langle\mathbf{x}_{i}-\mathbf{x}_{j}\right\rangle\right\} d V_{j}\right] .
$$

Consequently, the following expression is obtained when Eqs. (8) and (9) are added to Eq. (5):

$$
\begin{gathered}
\rho \ddot{\mathbf{u}}\left(\mathbf{x}_{i}, t\right)+\rho \alpha \dot{\mathbf{u}}\left(\mathbf{x}_{i}, t\right)=\sum_{j=1}^{N_{H}}\left\{\underline{\mathbf{T}}\left(\mathbf{x}_{i}, t\right)\left\langle\mathbf{x}_{j}-\mathbf{x}_{i}\right\rangle-\underline{\mathbf{T}}\left(\mathbf{x}_{j}, t\right)\left\langle\mathbf{x}_{i}-\mathbf{x}_{j}\right\rangle\right\} d V_{j} \\
+\beta \frac{d}{d t}\left[\sum_{j=1}^{N_{H}}\left\{\underline{\mathbf{T}}\left(\mathbf{x}_{i}, t\right)\left\langle\mathbf{x}_{j}-\mathbf{x}_{i}\right\rangle-\underline{\mathbf{T}}\left(\mathbf{x}_{j}, t\right)\left\langle\mathbf{x}_{i}-\mathbf{x}_{j}\right\rangle\right\} d V_{j}\right]+\mathbf{b}\left(\mathbf{x}_{\mathbf{i}}, \mathbf{t}\right)-\rho \ddot{\mathbf{u}}_{\mathbf{g}}\left(\mathbf{x}_{\mathbf{i}}, \mathbf{t}\right)
\end{gathered}
$$

To simplify discussions, two equations are defined as follows:

$$
\begin{array}{r}
\widetilde{\mathbf{T}}\left(\mathbf{x}_{i}, t\right):=\sum_{j=1}^{N_{H}}\left\{\underline{\mathbf{T}}\left(\mathbf{x}_{i}, t\right)\left\langle\mathbf{x}_{j}-\mathbf{x}_{i}\right\rangle-\underline{\mathbf{T}}\left(\mathbf{x}_{j}, t\right)\left\langle\mathbf{x}_{i}-\mathbf{x}_{j}\right\rangle\right\} d V_{j}, \\
\frac{d \underline{\widetilde{\mathbf{T}}}\left(\mathbf{x}_{i}, t\right)}{d t}:=\frac{d}{d t}\left[\sum_{j=1}^{N_{H}}\left\{\underline{\mathbf{T}}\left(\mathbf{x}_{i}, t\right)\left\langle\mathbf{x}_{j}-\mathbf{x}_{i}\right\rangle-\underline{\mathbf{T}}\left(\mathbf{x}_{j}, t\right)\left\langle\mathbf{x}_{i}-\mathbf{x}_{j}\right\rangle\right\} d V_{j}\right] .
\end{array}
$$

Substituting Eq. (11) into Eq. (10) leads to

$$
\rho \dot{\mathbf{u}}\left(\mathbf{x}_{i}, t\right)+\rho \alpha \dot{\mathbf{u}}\left(\mathbf{x}_{i}, t\right)=\underline{\widetilde{\mathbf{T}}}\left(\mathbf{x}_{i}, t\right)+\beta \frac{d \underline{\widetilde{\mathbf{T}}}\left(\mathbf{x}_{i}, t\right)}{d t}+b\left(\mathbf{x}_{i}, t\right)-\rho \dot{\mathbf{u}}_{g}\left(\mathbf{x}_{i}, t\right) .
$$




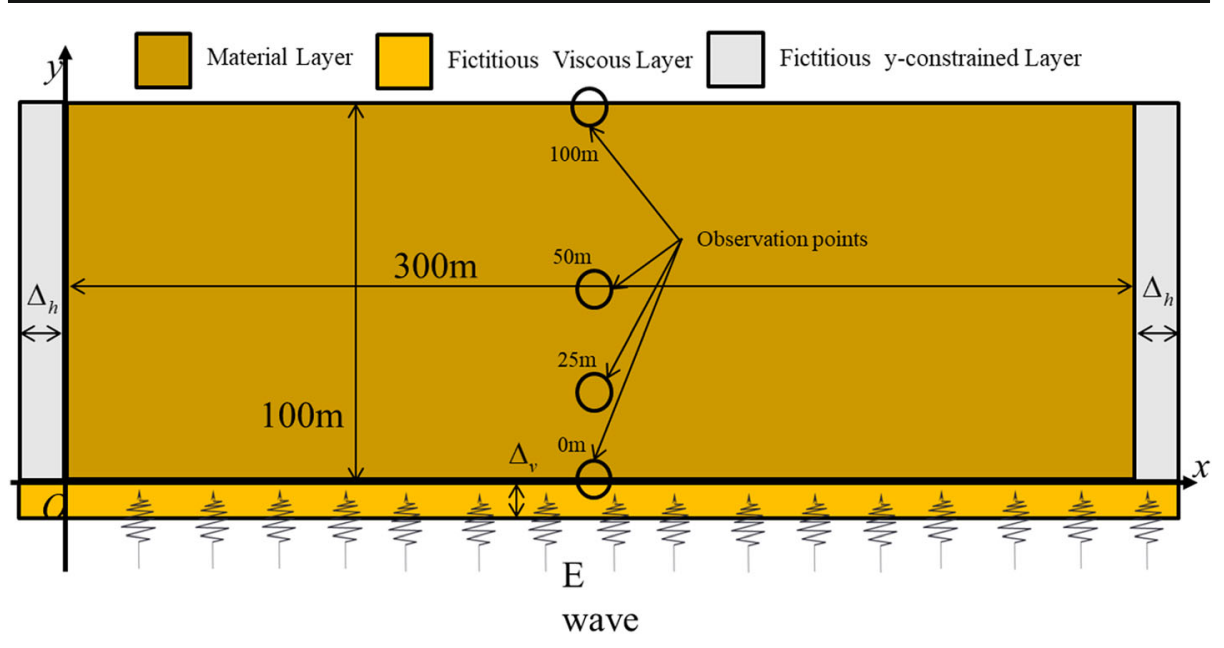

Fig. 4 The analysis model

\section{Implementation}

In this paper, we used a corrected surface effect [11] and a short-range force [14] in OSB-PD.

Figure 3 shows a definition of an analysis model with three layers. Here, the "Material layer" indicates the subject to the analysis, the "fictitious viscous layer," a viscous boundary layer that can express an incoming and outgoing seismic wave, and the "Fictitious yconstrained Layer," a fictitious layer to constrain displacement in the $y$-direction.

\subsection{Fictitious Viscous Boundary Layer}

A fictitious viscous boundary layer means that the same material as the fictitious viscous layer infinitely exists under the material layer. As such, using the fictitious viscous layer is possible to express an incoming/outgoing wave. If $\mathbf{u}_{g}\left(\mathbf{x}_{i}, t\right)$ in Eq. (12) is twice the input wave (i.e., well-known $2 \mathrm{E}$ wave), then only the E-wave enters the material layer.

For easy implementation, the layer is treated the same as a distributed pressure acting in materials [11]. From Eq. (1), a body force density vector can be expressed as,

$$
f_{\text {vis }, x}\left(\mathbf{x}_{i}, t\right)=-\frac{\rho V_{\mathrm{s}}}{\Delta_{v}} \dot{u}_{x}\left(\mathbf{x}_{i}, t\right)
$$

where $f_{\text {vis, } x}$ indicates a viscous force density of $x$-direction and $\Delta_{v}$ indicates the height of the fictitious viscous layer. When the fictitious viscous layer is of multiple layers, a non-viscous force is generated as the particles do not necessarily move uniformly. Therefore, in this paper, $\Delta_{v}$ is treated the same as particle distance, i.e., only one-line fictitious viscous layer is used. Furthermore, in the fictitious viscous layer, a displacement in the $y$-direction is constrained. To simplify discussions, we assumed that the material parameters ( $\rho$ and $V_{\mathrm{s}}$ ) of a fictitious viscous layer, are the same as those of the material layer. 

Table 1 Poisson's ratio, shear moduli, shear velocity, and arrival times of the seismic wave

\begin{tabular}{llllllllll}
\hline Frequency[Hz] & Poison's Ratio[-] & $\mu[\mathrm{MPa}]$ & $\mathrm{V}_{\mathrm{s}}[\mathrm{m} / \mathrm{s}]$ & $\mathrm{T} 1[\mathrm{~s}]$ & $\mathrm{T} 2[\mathrm{~s}]$ & $\mathrm{T} 3[\mathrm{~s}]$ & $\mathrm{T} 4[\mathrm{~s}]$ & $\mathrm{T} 5[\mathrm{~s}]$ & $\mathrm{T} 6[\mathrm{~s}]$ \\
\hline 0.5 & 0 & 56.00 & 174.7 & 0.143 & 0.286 & 0.573 & 0.859 & 1.002 & 1.145 \\
0.5 & 0.1 & 50.91 & 166.5 & 0.150 & 0.300 & 0.600 & 0.901 & 1.051 & 1.201 \\
0.5 & 0.25 & 44.80 & 156.2 & 0.160 & 0.320 & 0.640 & 0.960 & 1.120 & 1.280 \\
0.5 & 0.4 & 40.00 & 147.6 & 0.169 & 0.339 & 0.677 & 1.016 & 1.185 & 1.355 \\
\hline
\end{tabular}

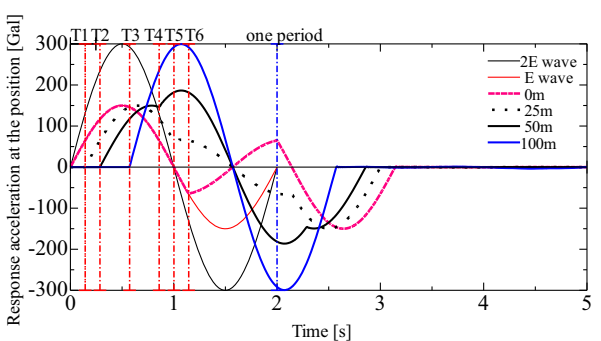

(a) OSB-PD

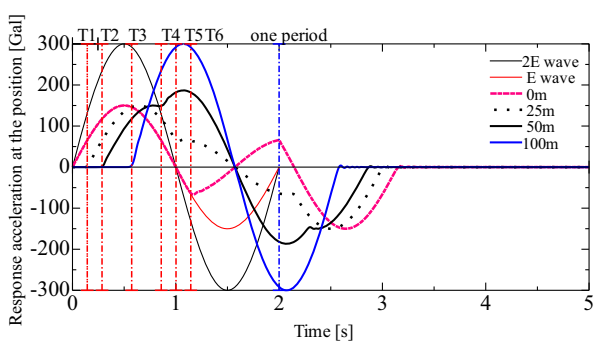

(b) FEM

Fig. 6 OSB-PD vs. FEM $(\nu=0.0,0.5 \mathrm{~Hz})$

\subsection{Fictitious Y-Constrained Layer $\Gamma_{u}^{v}$}

A fictitious y-constrained layer indicates the lateral boundaries of an analysis model that represents no vertical displacement at faraway places. Therefore, a displacement in the $y$ direction is constrained in the fictitious y-constrained layer as follows. Here, note that a displacement in the $x$-direction is free:

$$
\left\{\begin{array}{c}
\rho \ddot{u}_{x}\left(\mathbf{x}_{i}, t\right)=\widetilde{\mathbf{T}}_{x}-\rho \ddot{u}_{g, x}\left(\mathbf{x}_{i}, t\right) \\
u_{y}\left(\mathbf{x}_{i}, t\right)=0
\end{array}\right.
$$

where the subscripts $x$ and $y$ refer to the $x$ and $y$ components of these quantities, respectively.

\subsection{Time Integration}

Time integration of the OSB-PD equation of motion in Eq. (12) can be performed by using explicit forward difference technique as,

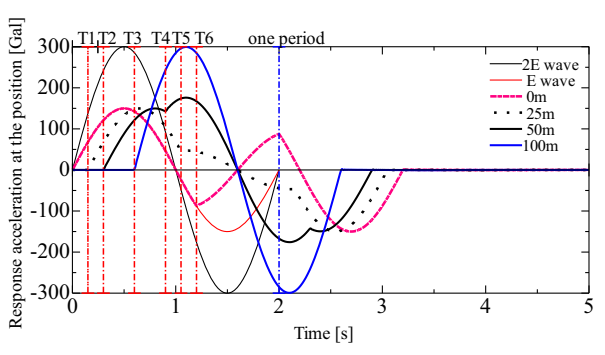

(a) OSB-PD

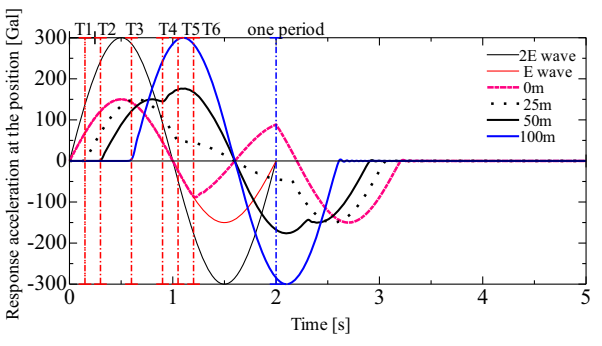

(b) FEM

Fig. 7 OSB-PD vs. FEM $(\nu=0.1,0.5 \mathrm{~Hz})$ 


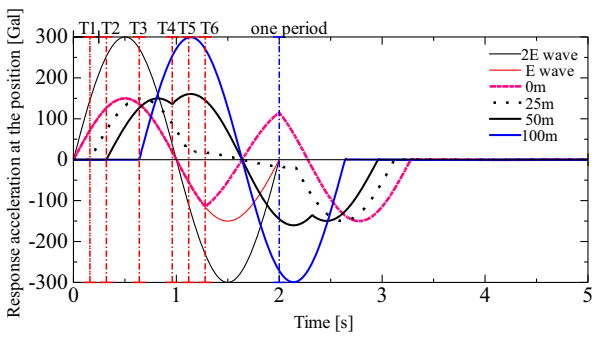

(a) OSB-PD

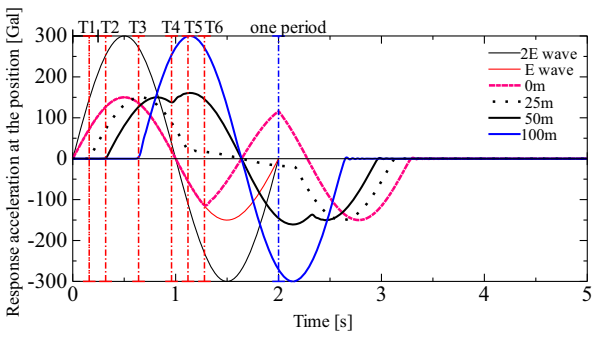

(b) FEM

Fig. 8 OSB-PD vs. $\operatorname{FEM}(\nu=0.25,0.5 \mathrm{~Hz})$

$$
\begin{aligned}
\dot{\mathbf{u}}\left(\mathbf{x}_{i}, t_{n+1}\right) & =\dot{\mathbf{u}}\left(\mathbf{x}_{i}, t_{n}\right)+\dot{\mathbf{u}}\left(\mathbf{x}_{i}, t_{n}\right) \Delta t \\
\mathbf{u}\left(\mathbf{x}_{\mathbf{i}}, \mathbf{t}_{\mathbf{n}+\mathbf{1}}\right) & =\mathbf{u}\left(\mathbf{x}_{\mathbf{i}}, \mathbf{t}_{\mathbf{n}}\right)+\dot{\mathbf{u}}\left(\mathbf{x}_{\mathbf{i}}, \mathbf{t}_{\mathbf{n}+\mathbf{1}}\right) \Delta \mathbf{t}
\end{aligned}
$$

where $n$ indicates the step number, $\Delta t$ is the time interval, and $t_{n+1}=t_{n}+\Delta t$. Substituting Eq. (15) into Eq. (12) leads to $\mathbf{u}\left(\mathbf{x}_{i}, t_{n+1}\right)$ as,

$$
\begin{gathered}
\dot{\mathbf{u}}\left(\mathbf{x}_{i}, t_{n+1}\right)=-\left\{\alpha \dot{\mathbf{u}}\left(\mathbf{x}_{i}, t_{n}\right)+\dot{\mathbf{u}}_{g}\left(\mathbf{x}_{i}, t_{n}\right)\right\}+\frac{1}{\rho}\left\{\underline{\widetilde{\mathbf{T}}}\left(\mathbf{x}_{i}, t_{n}\right)+\beta \frac{d \underline{\widetilde{\mathbf{T}}}\left(\mathbf{x}_{i}, t_{n}\right)}{d t}+b\left(\mathbf{x}_{i}, t_{n}\right)\right\}, \\
\text { where } \frac{d \widetilde{\mathbf{T}}\left(\mathbf{x}_{i}, t_{n}\right)}{d t} \approx \frac{\widetilde{\mathbf{T}}\left(\mathbf{x}_{i}, t_{n}\right)-\widetilde{\widetilde{\mathbf{T}}}\left(\mathbf{x}_{i}, t_{n-1}\right)}{\Delta t} .
\end{gathered}
$$

\section{Semi-Verification of OSB-PD}

We carried out simple calculations to semi-verify the proposed method. Firstly, a sine wave was applied onto a two-dimensional material layer through the fictitious viscous layer. Moreover, herein the proposed method was compared with a dynamic FEM. Specifically, the effect of Poisson's ratio, the input seismic wave number, and the Rayleigh damping components, were discussed following the calculation of response acceleration in both methods. Secondly, to semi-verify the proposed method the seismic response acceleration at the top of an analysis model, as calculated through the proposed method using an actual seismic acceleration time history, was compared with that calculated by dynamic FEM.

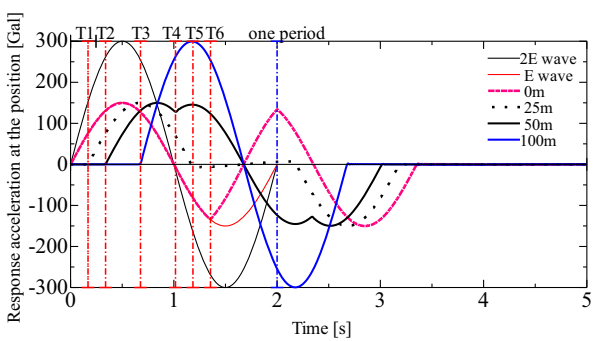

(a) OSB-PD

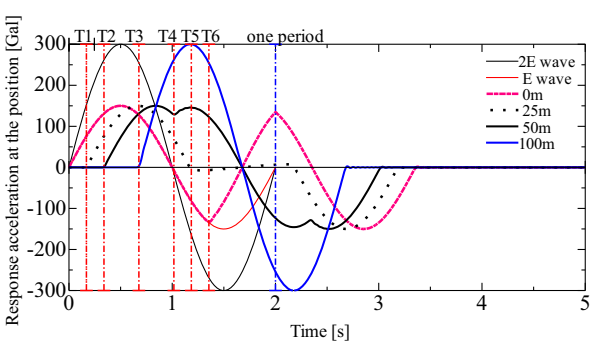

(b) FEM

Fig. 9 OSB-PD vs. FEM $(\nu=0.4,0.5 \mathrm{~Hz})$ 


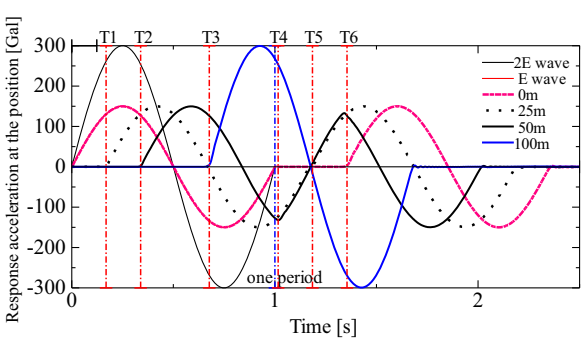

(a) OSB-PD

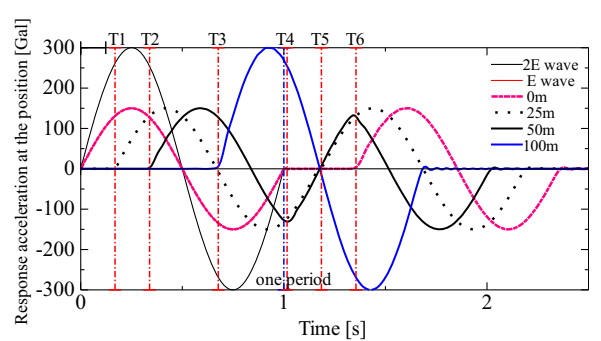

(b) FEM

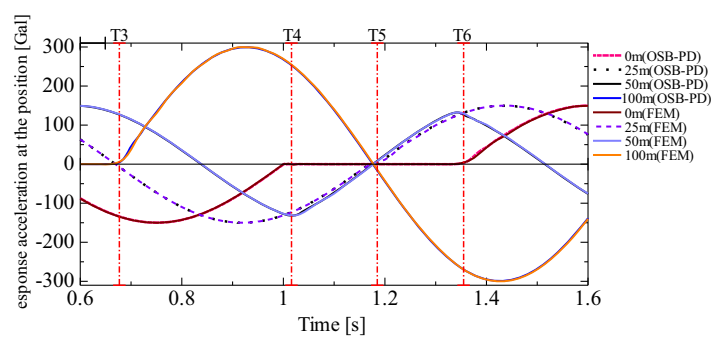

(c) Overlap of OSB-PD/FEM curves at $0.6-1.6 \mathrm{~s}$

Fig. 10 OSB-PD vs. FEM $(\nu=0.4,1.0 \mathrm{~Hz})$

\subsection{OSB-PD vs. FEM}

Figure 4 shows the analysis model created by using lattice particles. In the material layer, a total of 480,000 particles (i.e., 1200 lateral and 400 vertical particles) was considered, with an interval of $\Delta=0.25 \mathrm{~m}$ between each particle. A soft geo-material of approximately $V_{\mathrm{s}}=$

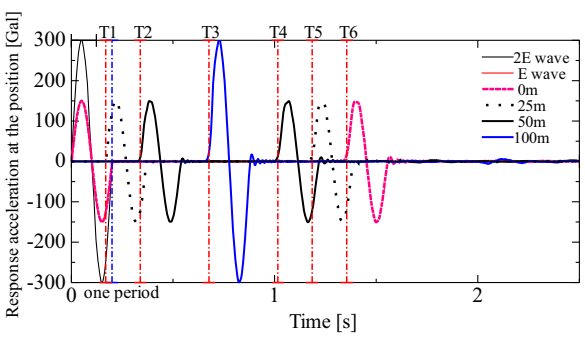

(a) OSB-PD

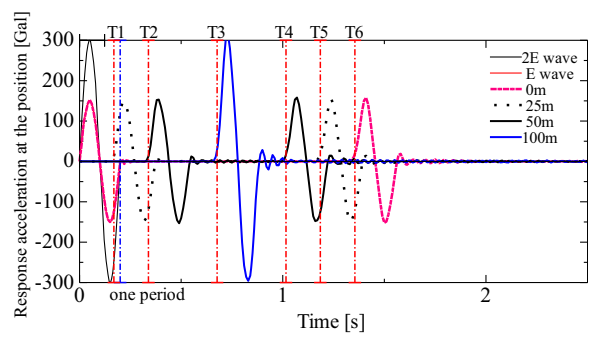

(b) FEM

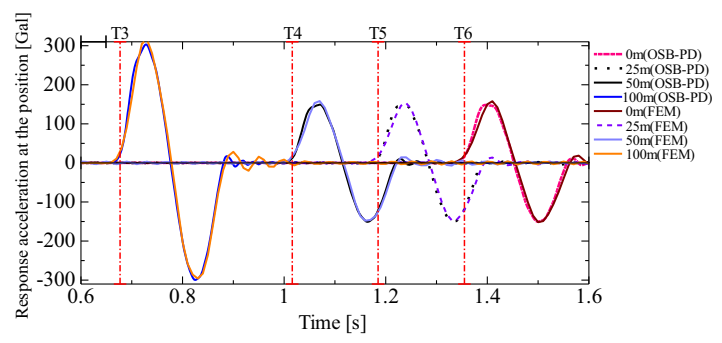

(c) Overlap of OSB-PD/FEM curves at 0.6-1.6s

Fig. 11 OSB-PD vs. FEM $(\nu=0.4,5.0 \mathrm{~Hz})$ 


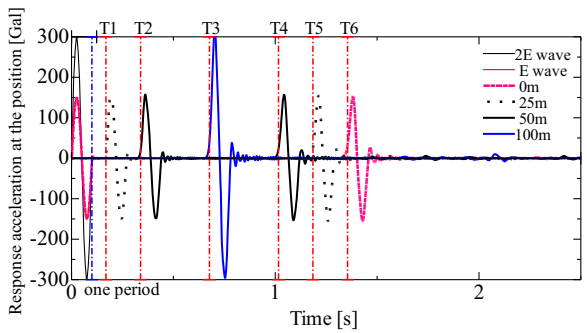

(a) OSB-PD

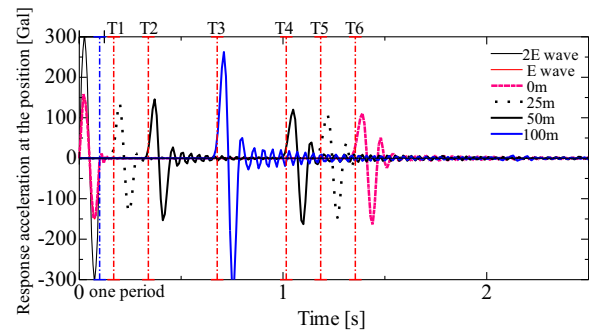

(b) FEM

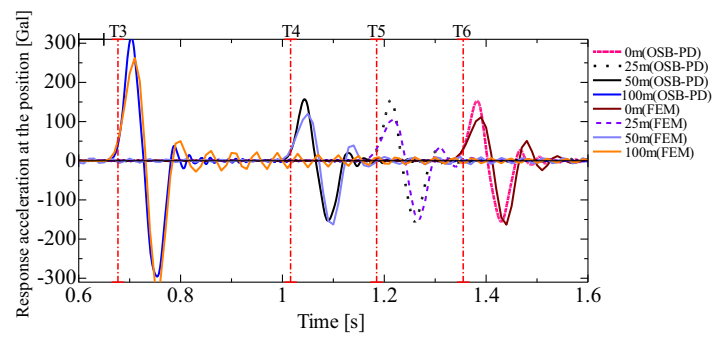

(c) Overlap of OSB-PD/FEM curves at $0.6-1.6 \mathrm{~s}$

Fig. 12 OSB-PD vs. FEM $(\nu=0.4,10 \mathrm{~Hz})$

$150 \mathrm{~m} / \mathrm{s}$ was selected herein. Thus, in the material layer and the fictitious $\mathrm{y}$-constrained layer, Young's modulus $E$ was set to $112 \mathrm{MPa}$ and the material density $\rho$ was set to $1835.5 \mathrm{~kg} / \mathrm{m}^{3}$. The height of the fictitious viscous layer $\Delta_{v}$ was set to $\Delta$ and the corresponding width $\Delta_{h}$ set to $5 \Delta$. "0 m," " $25 \mathrm{~m}$, , "50 m," and " $100 \mathrm{~m}$ " in Fig. 4 show observation points where calculated response accelerations are compared in both methods.

The input seismic wave (2E) $u_{g, x}$ is as below:

$$
u_{g, x}=\left\{\begin{array}{lc}
300 \sin (2 \pi f t) & (0 \leq t \leq T) \\
0 & \left(T<t \leq t_{\text {end }}\right)
\end{array} \quad[\mathrm{Gal}]\right.
$$

where $f=1 / T$ is the frequency $[\mathrm{Hz}]$ and $T$ is the period $[\mathrm{s}]$ and $t_{\text {end }}[\mathrm{s}]$ is the analysis end time. Note that only the E-wave was allowed to enter the material layer through the bottom of the material layer through the fictitious viscous layer. Herein and in the succeeding section, the maximum frequency of the input seismic wave used was $10 \mathrm{~Hz}$. Moreover, the OSB-PD parameter horizon $2 \delta$ was set to $2 \times 3.015 \Delta$ as approximately one-sixth of the wavelength $\left(V_{s}\right)$

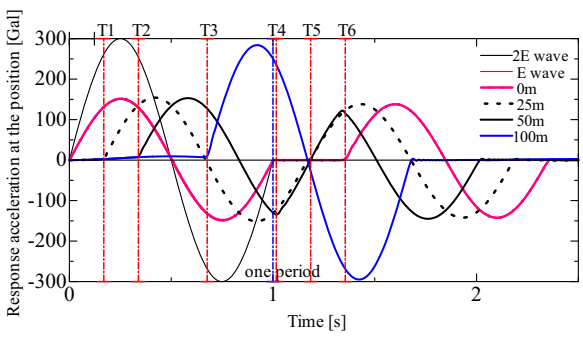

(a) OSB-PD

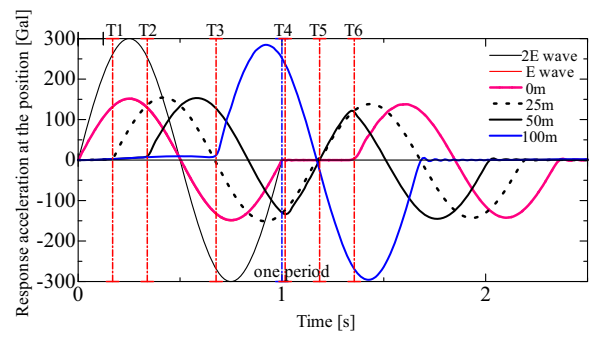

(b) FEM

Fig. 13 OSB-PD vs. FEM $(\alpha=0.1)$ 


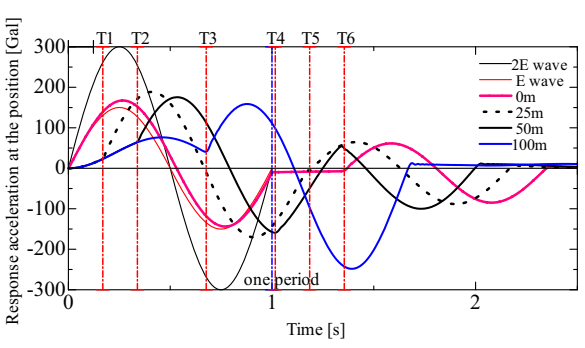

(a) OSB-PD

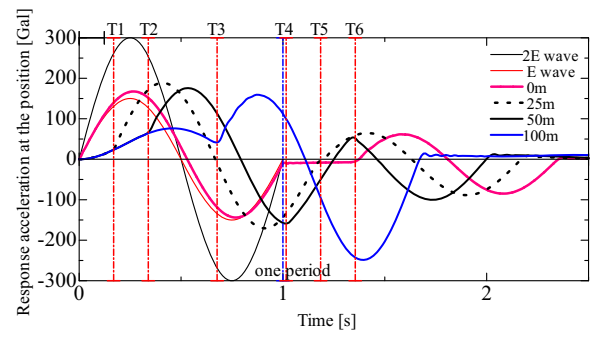

(b) FEM

Fig. 14 OSB-PD vs. FEM $(\alpha=1)$

f). Also, note that if the analysis model is large and the wavelength is short, enough degrees of freedom are needed to represent a single wavelength, which consequently leads to the number of particles being extremely large. To address this, shear velocity $V_{\mathrm{s}}$ was set to approximately $150 \mathrm{~m} / \mathrm{s}$, and the time interval $\Delta t[s]$ was ascertained with the expression,

$$
\Delta t=0.8 \sqrt{(2 \rho) /\left(\pi \delta^{2} b\right)}
$$

with reference to BB-PD [16].

Figure 5 displays the constructed FEM model via a 4-node isoparametric quadrilateral element. The FEM model has 2193 nodes and 2100 elements. The height of all elements was set to $2 \mathrm{~m}$, which was roughly one-sixth of the wavelength. The model size and the material parameters were the same as in the OSB-PD model. Moreover, the FEM model has yconstrained condition at the lateral edge, and a viscous boundary was used to express that only the E-wave should enter the FEM model through the bottom. Viscous boundary parameters were the same as in the PD model. More practically, the FEM calculation time interval $\Delta t[\mathrm{~s}]$ was set to $0.01 \mathrm{~s}$.

\subsubsection{Semi-Verification of the Fictitious Viscous Layer}

Seismic wave frequency $f$, analysis end time $t_{\text {end }}$, and Poisson's ratio was set to $0.5 \mathrm{~Hz}, 5 \mathrm{~s}$, and $(0,0.1,0.25,0.4)$, respectively. A list of all parameters used in the analysis is given in Table 1. The theoretical arrival times, $t=$ (arrival position) $/ V_{\mathrm{s}}$, were T1, T2, T3, T4, T5, and T6 for the respective seismic waves arrival positions $25,50,100,100+50$ (reflection), $100+75$ (reflection), and $100 \mathrm{~m}+100 \mathrm{~m}$ (reflection), respectively. Figures 6, 7, 8, and 9 illustrate a comparison between OSB-PD and FEM. In these figures, the expressions " $0 \mathrm{~m}$," "25 m,"

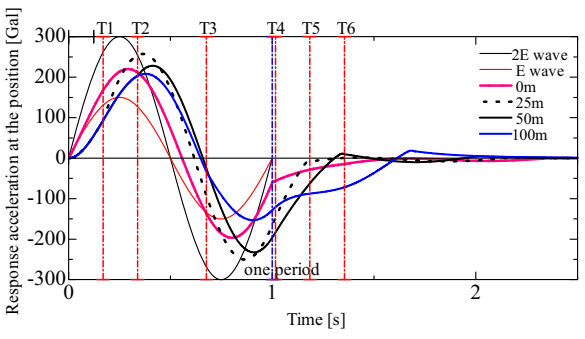

(a) OSB-PD

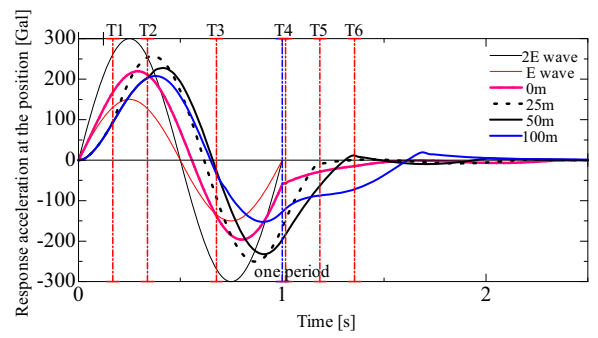

(b) FEM

Fig. 15 OSB-PD vs. FEM ( $\alpha=5)$ 


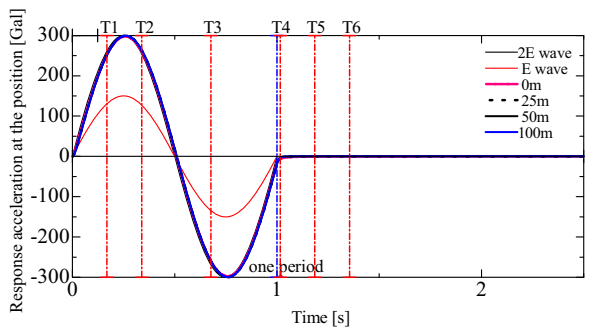

(a) OSB-PD

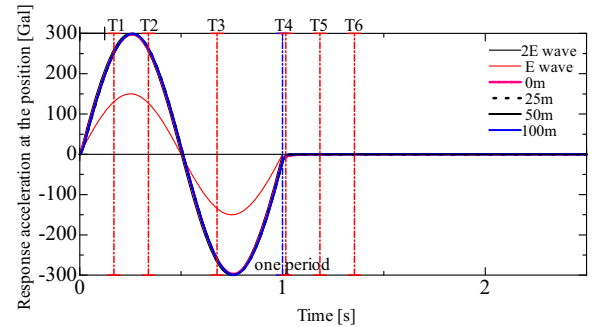

(b) FEM

Fig. 16 OSB-PD vs. FEM $(\alpha=100)$

"50 m," and " $100 \mathrm{~m}$ " correspond to the response acceleration at positions $0,25,50$, and $100 \mathrm{~m}$, respectively. Results for both methods showed good agreement qualitatively. On one hand, OSB-PD results at " $0 \mathrm{~m}$ " demonstrated that only E-wave entered the material layer until the reflected wave arrived at $0 \mathrm{~m}$. On the other hand, OSB-PD and FEM results at "100 m" showed the same response acceleration, with the maximum response acceleration of $300 \mathrm{Gal}$. Moreover, such results verified the well-known result in which the E-wave becomes $2 \mathrm{E}$-wave at a surface in the case of a no-damping analysis for a linear elastic material.

Furthermore, from the figures, the arrival times of OSB-PD and FEM were in good agreement with T1, T2, and T3. Similarly, the arrival times of OSB-PD and FEM, which include a reflection wave, agreed well with T4, T5, and T6. Upon arrival of the reflection wave (F-wave), E-wave becomes $\mathrm{E}+\mathrm{F}$ wave, and an inflection point is created on the response acceleration. Inflection points at T4, T5, and T6 were consistent in both results.

Thus, we clarified that the fictitious viscous layer in Eq. (13) exhibits the same function as the viscous boundary used in FEM.

\subsubsection{Semi-Verification of the Fictitious Viscous Layer for Several Frequencies}

Unlike in the preceding section where only a specific frequency was examined, herein several frequency sine waves were applied to the analysis model for evaluating the function of the fictitious viscous layer. Under such case, the Poisson's ratio was set to 0.4, while the input seismic wave (2E) considered and other parameters were as in the previous section. For a clearer demonstration of the analysis results, $t_{\text {end }}$ was set to $2.5 \mathrm{~s}$.

Figures 10, 11, and 12 depict the analysis results at response accelerations of 1, 5, and $10 \mathrm{~Hz}$, respectively. Qualitatively, results of OSB-PD and FEM agree well, as shown in Figs. 10 and 11. As in the previous section, all the OSB-PD results could express E-wave

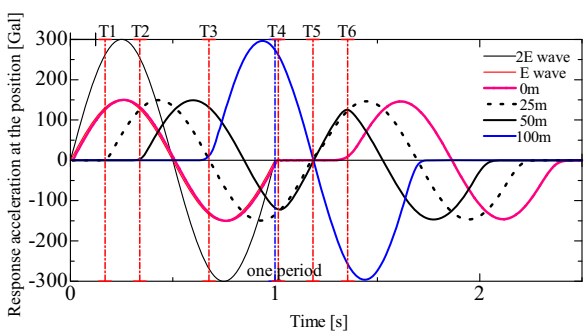

(a) OSB-PD

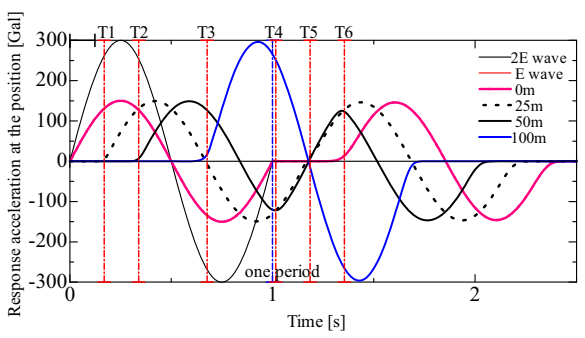

(b) FEM

Fig. 17 OSB-PD vs. FEM $(\beta=0.001)$ 


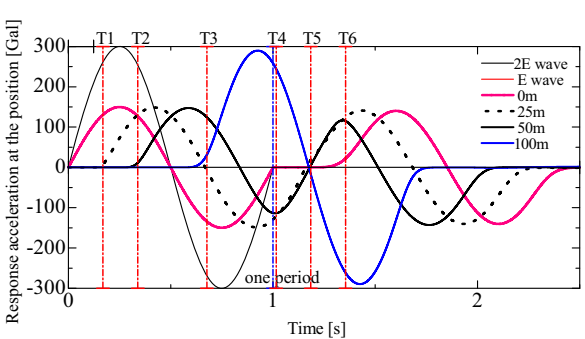

(a) OSB-PD

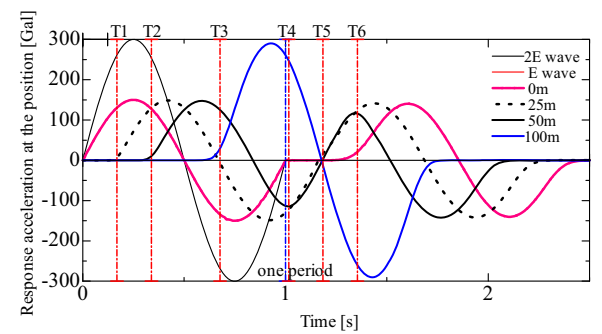

(b) FEM

Fig. 18 OSB-PD vs. FEM $(\beta=0.0025)$

transforming to $2 \mathrm{E}$-wave at $100 \mathrm{~m}$. However, a numerical vibration of response acceleration after T1 to T6 was confirmed in Fig. 12. Figures 10c, 11c and 12c indicate the overlap of OSB-PD and FEM curves at $t=0.6-1.6 \mathrm{~s}$. Figure $12 \mathrm{c}$ shows a qualitative difference between OSB-PD and FEM and the numerical vibration of response acceleration subsequent to T3. More specifically, the wavelength became short at high frequency of the input seismic wave, and several degrees of freedom were essentially needed to accurately represent the wavelength deformation. Consequently, the FEM result demonstrated that the E-wave could not be expressed into a $2 \mathrm{E}$-wave at $100 \mathrm{~m}$. When the degree of freedom was greater than that of FEM, the OSB-PD results showed relatively less numerical vibration and could then express the E-wave into a $2 \mathrm{E}$-wave at the same position. Although self-evident, a seismic response analysis should still be performed with careful attention to element height or particle distance.

The above results confirmed that the fictitious viscous layer can represent wave propagation and incoming/outgoing acceleration wave at several frequencies.

\subsubsection{Semi-Verification of Rayleigh Damping Components}

Under this section, we examined the effect of the Rayleigh damping components on the layers. To recall, Rayleigh damping components were used for the material layer and the fictitious yconstrained layer. The fictitious viscous layer had no Rayleigh damping components. The Rayleigh damping components $\alpha$ were $0.1,1,5$, and 100 , whereas the values for $\beta$ were 0.001 , 0.0025 , and 0.005 . Given that Eq. (9) is sensitive to the time interval, the calculation results normally diverge. To resolve this, the time interval should be smaller than in Eq. (18). Thus, the time interval was set to a value of the Eq. (18) divided by 10 , for the verification of $\beta$. In addition, in this section, seismic wave frequency $f$ was set to $1 \mathrm{~Hz}$.

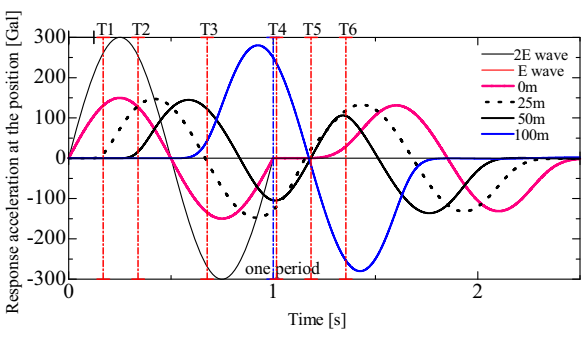

(a) OSB-PD

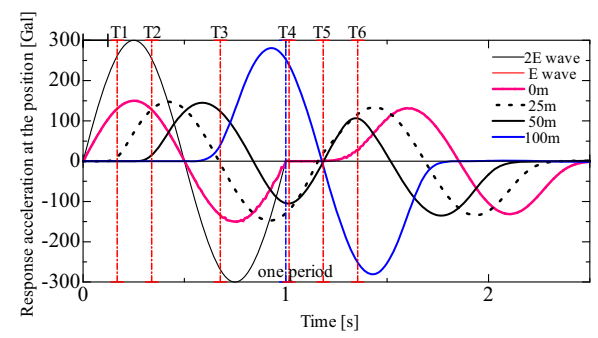

(b) FEM

Fig. 19 OSB-PD vs. FEM $(\beta=0.005)$ 


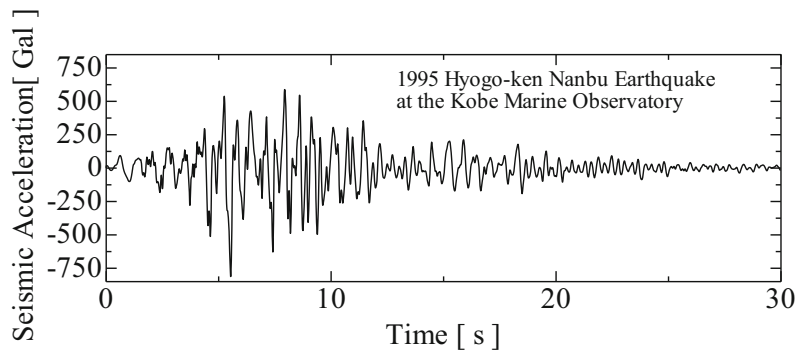

Fig. 20 Acceleration time history of the 1995 Hogyo-ken Nanbu Earthquake [28]

Moreover, Figs. 13, 14, 15, 16, 17, 18, and 19 demonstrate a good agreement qualitatively between the OSB-PD and FEM results. The effect of Rayleigh $\alpha$ was identical to that in the

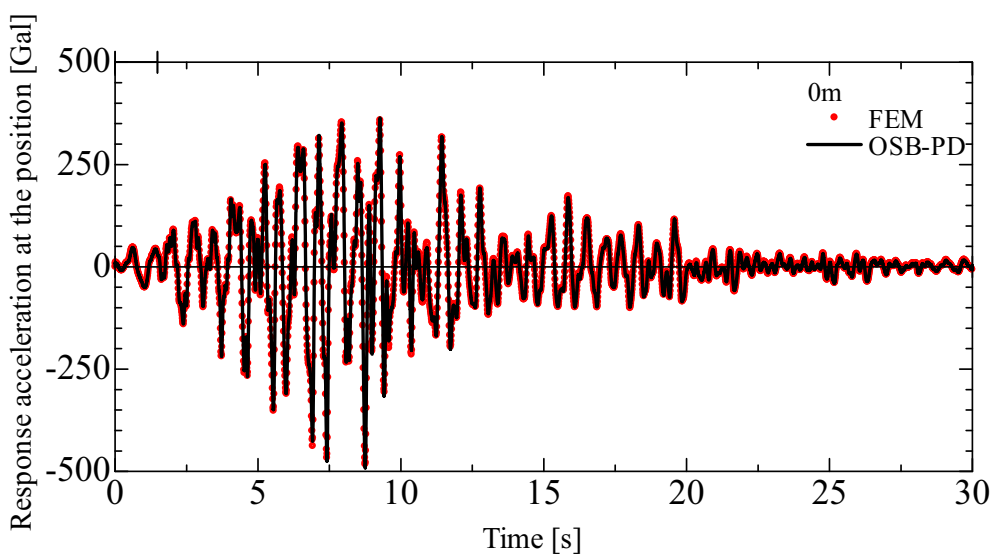

(a) $0 \mathrm{~m}$

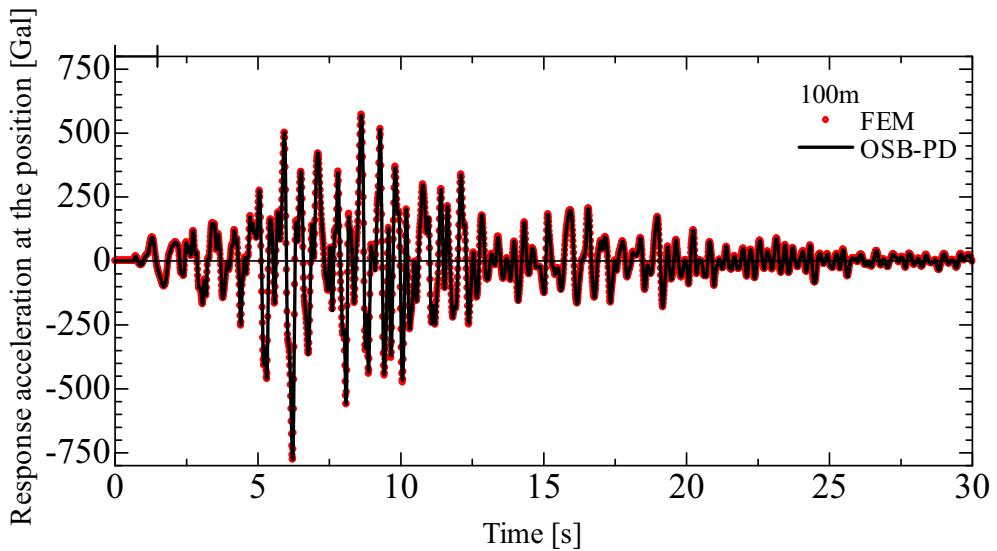

(b) $100 \mathrm{~m}$

Fig. 21 OSB-PD vs. FEM 


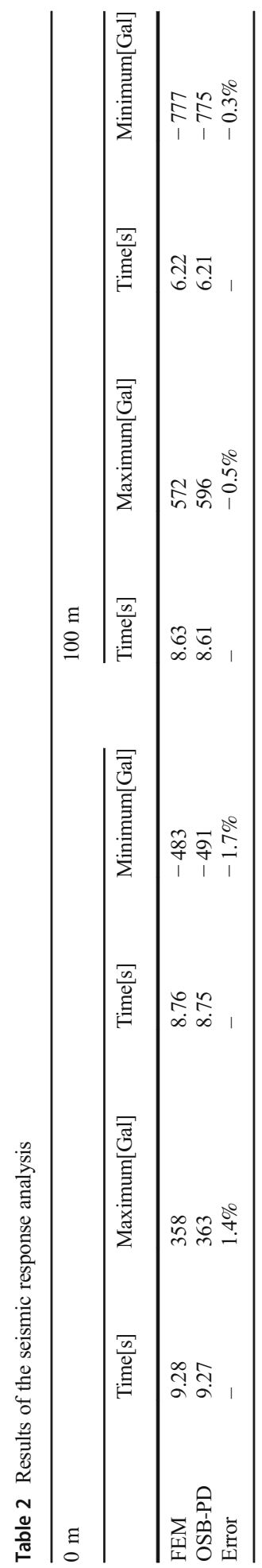


adaptive dynamic relaxation model by Madenci and Oterkus [16]. On one hand, when the value of $\alpha$ was high, the analysis result was close to the result of the quasi-static analysis. As such, no difference in time was observed, and the same response acceleration was obtained anywhere, as illustrated in Fig. 16. On the other hand, when the value of $\beta$ was high, the apparent hardness of the materials increased. Thus, it was shown in Figs. 17, 18, and 19 that the arrival times of the numerical result were different from the arrival times T1 to T6. In addition, due to the influence of the Rayleigh damping $\beta$, the E-wave did not transform to $2 \mathrm{E}$-wave at the $100 \mathrm{~m}$ position, as shown in Figs. 17, 18, and 19. This scenario was true for both analysis methods.

From the above results, we showed that the Rayleigh damping can be expressed by Eqs. (8) and (9).

\subsection{Seismic Acceleration}

Accordingly, to semi-verify our proposed method, we compared the response acceleration at the top of model, as calculated by the proposed method using an actual seismic acceleration time history, with that calculated by the dynamic FEM. Figure 20 shows the input seismic wave [28] from the 1995 Hogyo-ken Nanbu Earthquake (HNE), a direct-type (short-period) earthquake. In the event of HNE, the duration time was $30 \mathrm{~s}$, the sampling rate was $100 \mathrm{~Hz}$ (i.e., an observation time interval of $0.01 \mathrm{~s}$ ), and the maximum frequency $f_{\max }$ and minimum frequency $f_{\min }$ was 50 and $1 / 30 \mathrm{~Hz}$, respectively.

We employed the same analysis model as in the previous section. Young's modulus $E$ was set to $112 \mathrm{MPa}$ and the Poisson's ratio to 0.4 . The time interval was $6.79 \times 10^{-4} \mathrm{~s}$. Assuming $h=5 \%$ damping for all frequencies of the seismic wave, the corresponding Rayleigh damping components were as follows:

$$
\begin{aligned}
& \alpha=\frac{4 \pi h f_{\max } f_{\text {min }}\left(f_{\max }-f_{\min }\right)}{f_{\text {max }}^{2}-f_{\text {min }}^{2}}=0.02093 \\
& \beta=\frac{4 \pi h\left(f_{\text {max }}-f_{\text {min }}\right)}{f_{\text {max }}^{2}-f_{\text {min }}^{2}}=0.00032
\end{aligned}
$$

Figure 21 demonstrates that the results of OSB-PD and FEM consistently and qualitatively agreed with each other. In particular Fig. 21 a and b give the response acceleration at " 0 " and "100 m," respectively, whereas Table 2 provides the maximum/minimum response acceleration at these positions. Here, an error, mainly, the difference between OSB-PD and FEM divided by the value of FEM, was observed. Nevertheless, the resulting maximum error was $1.7 \%$, which meant that the OSB-PD and FEM results quantitatively agreed well.

Overall, our proposed method is applicable to seismic response analysis.

\section{Conclusion}

We formulated the OSB-PD, as well as the Rayleigh damping component, for a seismic response analysis on a viscous boundary surface. We semi-verified the method through a comparison of the dynamic response acceleration results from both our method and the dynamic FEM. The main findings of the study are as follows: 
1. Using several Poisson's ratio, i.e., several shear moduli, a qualitative agreement between the results of both methods was seen in all cases. Several frequencies were also examined. Under high frequency, a slight numerical vibration of the response acceleration was verified. However, the results for FEM and OSB-PD qualitatively agreed with each other in all cases. Therefore, the proposed viscous boundary layer is suitable to seismic response analysis.

2. Rayleigh damping was investigated in OSB-PD. On one hand, the mass-proportional damping behaved like the adaptive dynamic relaxation. On the other hand, the stiffnessproportional damping was defined by the time derivative of force density vectors. A few examples were examined, yielding a qualitative agreement between the FEM and OSB-PD results in all cases. Thus, Rayleigh damping can be expressed by the proposed method.

3. Seismic response acceleration at the top of the analysis model was calculated with the proposed method using an actual seismic acceleration time history and with the dynamic FEM. Both results agreed well with each other.

As future work, we will consider the viscous boundary layer and Rayleigh damping to clarify the tensile failure and sliding failure phenomenon of soil embankment caused by an earthquake.

Funding Information This work was supported by JSPS KAKENHI, Grant Number JP 19 K04957.

\section{Compliance with Ethical Standards}

Conflict of Interest The authors declare that they have no conflict of interest.

Open Access This article is licensed under a Creative Commons Attribution 4.0 International License, which permits use, sharing, adaptation, distribution and reproduction in any medium or format, as long as you give appropriate credit to the original author(s) and the source, provide a link to the Creative Commons licence, and indicate if changes were made. The images or other third party material in this article are included in the article's Creative Commons licence, unless indicated otherwise in a credit line to the material. If material is not included in the article's Creative Commons licence and your intended use is not permitted by statutory regulation or exceeds the permitted use, you will need to obtain permission directly from the copyright holder. To view a copy of this licence, visit http://creativecommons.org/licenses/by/4.0/.

\section{References}

1. Yoshikawa T, Noda T, Kodaka T, Takaine T (2016) Analysis of the effect of groundwater level on the seismic behavior of an unsaturated embankment on clayey ground. Soil Dyn Earthq Eng 85:217-230

2. Nonaka T, Yamada S, Noda T (2017) Verification of a macro-element method in the numerical simulation of the pore water pressure dissipation method-a case study on a liquefaction countermeasure with vertical drains under an embankment. Soils Found 57(3):472-487

3. Oka F, Tsai P, Kimoto S, Kato R (2012) Damage patterns of river embankments due to the 2011 off the Pacific Coast of Tohoku Earthquake and a numerical modeling of the deformation of river embankments with a clayey subsoil layer. Soils Found 52(5):890-909

4. Morishima N (2018) Effective stress analysis of river dikes during the 2011 East Japan Earthquake. In: Iai S (ed) Developments in earthquake geotechnics. Geotechnical, Geological and Earthquake Engineering, p 43. https://doi.org/10.1007/978-3-319-62069-5_12

5. Shimbo T (2017) Development and application of a dynamic XFEM for the seismic residual displacement analysis of an embankment. Soils Found 57(3):357-370

6. Yong Z, Pizhong Q (2019) A new bond failure criterion for ordinary state-based peridynamic mode II fracture analysis. Int J Fract 215(1-2):105-128 
7. Ren H, Zhuang X, Rabczuk T (2016) A new peridynamic formulation with shear deformation for elastic solid. J Micromech Mol Physics 1(2):1650009

8. Wang Y, Zhou X, Xu X (2016) Numerical simulation of propagation and coalescence of flaws in rock materials under compressive loads using the extended non-ordinary state-based peridynamics, Engineering Fracture Mechanics. 163:248-273

9. Silling S (2000) Reformulation of elasticity theory for discontinuities and long-range forces. J Mech Physics Solids 48(1):175-209

10. Silling SA, Epton M, Weckner O, Xu J, Askari A (2007) Peridynamics states and constitutive modeling. J Elast 88(2):151-184

11. Madenci E, Oterkus S (2016) Ordinary state-based peridynamics for plastic deformation according to von Mises yield criteria with isotropic hardening. J Mech Physics of Solids 86:192-219

12. Chen W, Zhu F, Zhao J, Li S, Wang G (2018) Peridynamics-based fracture animation for elastoplastic solids. Comp Graph Forum 37(1):112-124

13. Siavash N (2018) Improved state-based peridynamic lattice model including elasticity, plasticity and damage. Comput Model Eng Sci 116(3):323-347

14. Macek RW, Silling SA (2007) Peridynamics via finite element analysis. Finite Elem Anal Des 43(15):11691178

15. Shojaei A, Mudric T, Zaccariotto M, Galvanetto U (2016) A coupled meshless finite point/Peridynamic method for 2D dynamic fracture analysis. Int J Mech Sci 119:419-431

16. Zaccariotto M, Mudric T, Tomasi D, Shojaei A, Galvanetto U (2018) Coupling of FEM meshes with Peridynamic grids. Comput Methods Appl Mech Eng 330:471-497

17. Katiyar A, Foster JT, Ouchi H, Sharma MM (2014) A peridynamic formulation of pressure driven convective fluid transport in porous media. J Comput Phys 261:209-229

18. Ouchi H, Katiyar A, York J, Foster JT, Sharma MM (2015) A fully coupled porous flow and geomechanics model for fluid driven cracks: a peridynamics approach. Comput Mech 55(3):561-576

19. Ouchi H (2016). Development of peridynamics-based hydraulic fracturing model for fracture growth in heterogeneous reservoirs (Doctoral dissertation)

20. Nadimi S, Miscovic I, Mclennan J (2016) A 3D peridynamic simulation of hydraulic fracture process in a heterogeneous medium. J Pet Sci Eng 145:444-452

21. Dong Y, Xiaoqiao H, Shenghui Y, Xuefeng L (2019) An improved ordinary state-based peridynamic model for cohesive crack growth in quasi-brittle materials. Int J Mech Sci 153:402-415

22. Madenci E, Oterkus E (2014) Peridynamic theory and its applications, 17th edn. Springer, Berlin

23. Diehl P, Prudhomme S, Lévesque M (2019) A review of benchmark experiments for the validation of peridynamics models. J Peridynamics Nonlocal Model. https://oi.org/10.1007/s42102-018-0004-X

24. Duanfeng H, Yiheng Z, Qing W, Wei L, Bin J (2018) The review of the bond-based peridynamics modeling. J Micromech Mol Physics. https://doi.org/10.1142/S2424913018300013

25. Hafezi MH, Alebrahim R, Kundu T (2017) Peri-ultrasound for modeling linear and nonlinear ultrasonic response. Ultrasonics 80:47-57

26. Wang L, Chen Y, Xu J, Wang J (2017) Transmitting boundary conditions for 1D peridynamics. Int J Numer Methods Eng 110(4):379-400

27. Lysmer J, Kuhlemeyer RL (1969) Finite dynamic model for infinite media. J Eng Mech Div 95(4):859-876

28. Japanese Road Association: Specifications for highway bridges, Part V: Seismic design, 2017. (In Japanese) 